\title{
RAF autoinhibition and 14-3-3 proteins promote paradoxical activation
}

\author{
Gaurav Mendiratta, Thomas McFall and Edward C. Stites
}

Integrative Biology Laboratory, Salk Institute for Biological Studies, La Jolla, CA, USA.

\begin{abstract}
:
RAF kinase inhibitors can actually increase RAF kinase signaling. This process, which is commonly referred to as "paradoxical activation" (PA), is incompletely understood. RAF kinases are regulated by autoinhibitory conformational changes, and the role of these conformational changes in PA is unclear. Our mathematical investigations find that PA can result from a dynamical equilibrium between autoinhibited and non-autoinhibited forms of RAF, along with the RAF inhibitor stabilizing the non-autoinhibited form. We also investigate whether PA is influenced by 14-3-3 proteins, which can both stabilize RAF autoinhibition and RAF dimerization. Using both computational and experimental methods we demonstrate that 14-3-3 proteins potentiate PA. Third generation RAF inhibitors normally display minimal to no PA. Our mathematical modeling led us to hypothesize that increased 14-3-3 expression should also amplify PA for these agents. Subsequent experiments support our hypothesis and show that 14-3-3 overexpression increases PA in these third generation RAF inhibitors, effectively "breaking" these "paradox breakers" and pan-RAF inhibitors. We have therefore created and experimentally validated a robust mechanism for PA based solely on equilibrium dynamics of canonical interactions in RAF signaling.
\end{abstract}




\section{Introduction}

The RAS/RAF pathway plays essential roles in human cancer. Proliferation signals generated by transmembrane receptors signal through RAS GTPases to the RAF kinases that initiate the RAF/MEK/ERK Mitogen Activated Protein Kinase (MAPK) cascade. Mutations within this pathway are very common in cancer (1). Multiple drugs that target this pathway have been developed to inhibit the RAF kinases (2-5) and these agents have proven clinically valuable for melanoma (6-8) and colorectal cancer (9-11). Additionally, RAF inhibitors appear promising for other BRAF mutant cancers (12-14).

As the RAF kinases (BRAF, CRAF, and ARAF) are key conduits of signals from the RAS (KRAS, NRAS, and HRAS) GTPases, it was originally hoped that RAF inhibitors would be able to block the transmission of RAS signals (Figure 1A). However, RAF inhibitors were instead unexpectedly found to amplify RAS signals through a process that is commonly referred to as "paradoxical activation" (PA) (Figure 1B) (15-17). Despite numerous studies, the mechanisms driving PA are still not fully understood $(4,18,19)$. Mechanistically, two behaviors are commonly implicated as contributing to the phenomenon of PA. First, some RAF inhibitors have been shown to result in an increased level of RAF dimerization (Figure 1C) $(17,18,20$, 21). Second, many RAF inhibitors do not appear capable of binding to both protomers of a RAF dimer equally well $(4,18,19)$. This may be due to differences in how well the drug binds to the first and second sites within a RAF dimer. Such differences may arise because the compound binds with different affinities to BRAF, CRAF, and/or $\operatorname{ARAF}(2,4,18)$ and RAF signals propagate through hetero- and homodimers $(15-17,22)$. Alternatively, there may also be negative cooperativity (also referred to as negative allostery) in which the binding of a RAF inhibitor to one protomer of a RAF dimer reduces the affinity of the second protomer for RAF inhibitor (Figure 1D) $(18,19,23)$. As only one protomer in a RAF dimer need be signaling competent for RAF signaling to propagate $(15,24,25)$, negative cooperativity can result in a reduced ability to fully inhibit RAF signaling. However, the regulation of RAF kinase activation is complex with multiple regulatory steps $(18,24,26,27)$, and other processes have been described to play a role in PA $(15,28-31)$.

Among the regulatory steps are RAF conformational changes $(21,26,27)$. In the "autoinhibited" form, associations between the N-terminus of RAF and the kinase domain maintain RAF in a non-active form that does not dimerize (Figure 1E) $(26,32)$. In the "non- 
autoinhibited" form, the kinase domain is no longer occluded, and other regulatory mechanism that contribute to full RAF kinase activation may occur, such as kinase domain conformational changes and dimerization $(20,26)$. Recent experimental work observes that RAF inhibitors tend to promote a net transition to the non-autoinhibited conformation that is bound to RAS-GTP (19, 21). It has been suggested that this biasing to the non-autoinhibited state may contribute to PA (21).

Here, we report our analysis of the contribution of RAF autoinhibitory conformation changes on PA and the experimental testing of a startling insight from the modeling that has important clinical implications. On the theoretical side, we developed a series of mathematical models that describe key regulatory steps that have been implicated or suggested to play a role in RAF signaling. We developed these models to follow biochemical principles and thermodynamics, and then utilized the models to derive the behaviors that would logically follow from these principles and mechanisms. Our modeling reveals that RAF autoinhibitory conformational changes alone can be sufficient to drive PA. We believe this is the key process that has been underappreciated and will help resolve ongoing uncertainties with regards to PA. For example, we find that autoinhibitory conformational dynamics and their perturbation by drugs can explain the magnitudes of PA that have been observed. We extended our model to include the roles of 14-3-3 proteins in stabilizing RAF in the autoinhibitory state and also in stabilizing RAF dimers $(33,34)$, both of which have generated recent attention due to new cryoEM structures of these complexes $(30,35)$. We mathematically find 14-3-3 can further potentiate PA, and that this effect increases with increasing levels of 14-3-3 expression. This led us to hypothesize that drugs developed to display minimal to no PA under standard cellular conditions could be compromised by increased PA due to amplified 14-3-3 expression. Our experiments confirm this, revealing that 14-3-3 overexpression can amplify PA for existing RAF inhibitors and can even create PA for third generation RAF inhibitors that were designed not to display PA.

\section{Results}

\section{Mathematical modeling of RAF autoinhibition}

We developed a mathematical model to study whether the stabilization of RAF in its nonautoinhibited state by RAF inhibitors may be sufficient to generate PA. A mathematical model 
of biochemical processes allows one to rigorously analyze what behaviors are possible for a given set of reaction mechanisms and can lead to non-obvious hypotheses for further experimental testing (36-38). Our first mathematical model allows RAF to adopt two different conformations: one of which is autoinhibited and can neither dimerize nor bind drug, the other of which is non-autoinhibited and can bind drug and/or dimerize (20). Drug-bound RAF is assumed to only be able to transition back to an autoinhibited state after any bound drug has dissociated. A complete mechanism is portrayed in Figure 2A; this mechanism was implemented as an ordinary differential equation model. We derived closed-form, analytic expressions for the steady-state solution for this system using the principal of detailed balance $(39,40)$. We evaluated RAF signaling activity as a function of RAF inhibitor abundance, and we found that our proposed mechanism of RAF conformational autoinhibition and interaction with RAF inhibitors was sufficient to create PA (Figure 2B).

\section{Paradoxical activation reflects a shifting balance of signaling complexes}

To understand how PA arises from this mechanism, we considered the proportion of RAF in each of its possible states: (a) autoinhibited RAF monomer, (b) non-autoinhibited RAF monomer that is not bound to drug, (c) non-autoinhibited monomer that is bound to drug, (d) RAF dimer with no drug bound, (e) RAF dimer with one of two kinase domains bound to drug, (f) RAF dimer with both kinase domains bound to drug. We considered the total amount of kinase activity to be the number of RAF protomers within a dimer that are not bound to drug.

Before drug is given, a significant fraction of RAF is autoinhibited and there are low levels of non-autoinhibited RAF and RAF dimers. As RAF inhibitor levels increase, the level of autoinhibited RAF progressively declines (Figure 2C). Non-autoinhibited RAF distributes between drug-bound monomeric and dimeric forms while the unbound monomeric form maintains equilibrium with the auto-inhibited RAF (Figure 2C). The increased quantity of RAF dimers reflects the increased pool of RAF proteins that are non-autoinhibited and therefore capable of dimerization. The resulting shift in dynamical equilibrium maintains inhibitory response of dimer populations unbound to the drug (Figure 2C). However, the same effect causes a drug-dependent rise in RAF dimers bound to drug in one site (Figure 2C) and therefore total RAF kinase activation that accounts for PA. PA dose responses also display an eventual reduction in RAF kinase activity. The quantity of drug-bound RAF monomer and doubly-drug- 
bound RAF dimer progressively increases to saturation as a function of the drug amount (Figure 2C).

\section{Paradoxical activation is a robust outcome of conformational autoinhibition}

We can show analytically that this mechanism, as implemented in our model, is sufficient to create PA. Our analysis yields algebraic expressions that define the conditions necessary for PA to occur by this mechanism: by substituting biophysical numbers into these expressions we can predict PA for a wide range of RAF concentration values (Fig. 3). We can also show that simpler mechanisms cannot display PA, such as one without the autoinhibitory conformational changes and one without dimerization.

\section{4-3-3 proteins increase the magnitude of paradoxical activation}

We next asked how proteins that stabilize the autoinhibited form of RAF might influence PA. 14-3-3 proteins are phosphoserine binding proteins that interact with a large number of proteins and contribute to a wide variety of cellular processes (41). The RAF kinases are wellknown binding partners for 14-3-3 (42). 14-3-3 proteins bind and stabilize the autoinhibited form of $\operatorname{BRAF}(33,34,43)$, and a recent cryo-EM structure is available for the complex between 14-3-3 and autoinhibited BRAF (35). However, 14-3-3 also stabilizes the RAF dimers (25, 33, 34) and a recent cryo-EM structure illuminates the structure of dimerized RAF bound to 14-3-3 (30). We therefore extended our model to include both of these sets of reactions (Figure 4A) and we then mathematically investigated how they may impact PA.

Our analysis suggests that 14-3-3 proteins should significantly potentiate PA (Figure 4B). To evaluate whether this was due to either stabilization of the autoinhibited form, to stabilization of dimers, or both we considered simplified models that included either autoinhibited form stabilization by 14-3-3 (without dimer stabilization by 14-3-3), or dimer stabilization by 14-3-3 (without autoinhibited form stabilization by 14-3-3). We found that autoinhibited form stabilization potentiated PA to a greater extent than the case where both roles of 14-3-3 were considered (Figure 4B). We also found that dimerization stabilization alone not only failed to potentiate PA but could also reduce it (Figure 4B), thus suggesting that autoinhibited form stabilization is the dominant mechanism by which 14-3-3 proteins will potentiate PA. 


\section{4-3-3 expression expands the range of conditions in which paradoxical activation occurs}

We next analyzed whether the presence of 14-3-3 proteins also changes the conditions under which PA could occur. Our analysis found that increasing levels of 14-3-3 expression result in increased magnitudes of PA and a wider range of drug conditions where the PA effect is observed (Figure 4C). We also found that PA is an even more robust outcome of RAF autoinhibitory regulation when the roles of 14-3-3 proteins are also considered, in that the set of parameters for which PA can result is larger than the set of parameters for which PA can result when no 14-3-3 proteins present (Fig. 5). Importantly, this analysis also reveals that increasing the level of 14-3-3 protein expression can result in PA under conditions where it would not otherwise have occurred.

\section{Experiments: 14-3-3 overexpression amplifies paradoxical activation}

We experimentally tested the model-based hypothesis that increased expression of 14-3-3 proteins can potentiate PA. We transfected 14-3-3 zeta into RAS mutant cells and confirmed 143-3 transfection by immunoblot (Figure S1) and then performed a vemurafenib dose response, using ERK phosphorylation as a readout of RAF kinase activity. We first utilized SK-MEL-2 melanoma cells, which are BRAF WT and harbor an oncogenic NRAS Q61R mutation (Figure 6A). We observed significant widening of the PA response in 14-3-3 transfected cells, but not the mock transfected cells, consistent with model predictions. This widening is consistent with the behavior predicted by our mathematical model.

We performed the same experiments in SW48 colorectal cancer cells that had been engineered to have the KRAS G13D mutant to evaluate the generality of our observation. In these SW48 cells treated with vemurafenib, we also observed that increased 14-3-3 expression resulted in PA that spans a wider range of drug concentrations, again consistent with the behavior predicted by our mathematical model (Figure S2).

\section{Experiments: 14-3-3 overexpression increases paradoxical activation in third generation RAF}

\section{inhibitors}

Ongoing work in RAF inhibitor drug development aims to develop RAF inhibitors where PA does not limit their utility (3-5). These third generation RAF inhibitors should be less prone 
to dimerization induced resistance, may cause less side effects, and some may even be useful for cancers with a RAS mutation. One strategy has been to develop drugs that target both monomeric and dimeric RAF kinases with high affinity and with no or minimal negative cooperativity $(4,5)$. These "pan-RAF inhibitors," such as LY3009210 and TAK-632, are described to have less PA and for the inhibitory response to occur at a lower quantity of drug (4, 5). However, our modeling suggests that 14-3-3 overexpression can stabilize autoinhibitory conformational dynamics to potentiate PA. We therefore hypothesized that 14-3-3 overexpression would counteract the favorable PA profile of pan-RAF third generation RAF inhibitors.

We tested this hypothesis in SK-MEL-2 cells transfected with 14-3-3 for both LY3009210 and TAK-632. 14-3-3 transfection was confirmed by immunoblotting (Figure S1). In mock transfected cells, we detected PA for both LY3009210 (Figure 6B) and TAK-632 (Figure 6C) in parental SK-MEL-2 cells. This is consistent with studies of these same inhibitors in this same cell line in a previous work (18). Of note, we observed the window for PA to occur at orders of magnitude lower concentrations for these third generation RAF inhibitors than we observed for vemurafenib.

In SK-MEL-2 cells transfected with 14-3-3 zeta, we found much more robust PA for both LY3009210 (Figure 6B) and TAK-632 (Figure 6C). For both inhibitors, 14-3-3 zeta overexpression resulted in a widening of the PA effect so that it was observed for over two orders of magnitude more concentrations of drug treatment. Thus, we find that the favorable PA profile of the "pan-RAF" third generation RAF inhibitors is less favorable when 14-3-3 is expressed at a higher level.

\section{Experiments: 14-3-3 overexpression creates paradoxical activation in "Paradox Breaker" third generation RAF inhibitor}

Another class of third generation inhibitors, the "paradox breakers," includes PLX8394. The strategy used to develop the paradox breakers involved finding compounds with a large separation between the dose of inhibitor that inhibits $50 \%$ of the pERK signal within a BRAF V600E mutant cell and the dose that promotes $50 \%$ of the maximal increase in pERK within a RAS mutant cell (3). Based on our analysis of autoinhibitory-driven PA and the role of 14-3-3 
proteins, we hypothesized that increased 14-3-3 expression could result in large magnitude PA for this paradox breaker in conditions where it otherwise would not display PA.

To test our hypothesis, we transfected SK-MEL-2 cells to express additional 14-3-3 proteins (Figure S1). We then treated these cells with a wide range of doses of PLX8394 (Figure 6D). As expected $(3,18,44)$, we did not detect PA when we looked at the dose response of PLX8394 in the mock transfected, parental SK-MEL-2. However, with elevated 143-3 expression we observed significant PA, consistent with our hypothesis.

\section{Discussion}

Overall, our mathematical analysis suggests that the conformational regulation of RAF kinase activity combined with inhibitor dependent dimerization is a critical mechanism that drives PA. Although autoinhibition has been recognized as an important component of the entire PA phenomenon $(15,21)$ it appears to have been underappreciated as a mechanism capable of driving PA; for example, it has neither been discussed as a motivation for the development of third generation RAF inhibitors (3-5) nor been included in recent mathematical analyses of PA $(40,45)$. This is notable, as both drug development and mathematical analyses pay considerable attention to conformations within the kinase domain (i.e. whether the alpha-C helix and DFG motif are in the "in" or "out" conformations) $(3-5,40,45)$. That suggests the concept of structural and conformational factors on PA is not foreign to drug developers and theoretical biologists, but that they may have limited their attention to the small changes in the kinase domain that have dominated the recent literature at the expense of also considering the large, autoinhibitory, conformational changes.

One recent study highlights the importance of RAF conformational change regulation and demonstrates clearly RAF kinase inhibitors promoting a net change to dimerized RAF in the active conformation and suggests that this plays an important role in PA (21); however, the process described in that work does not clearly define the mechanism from which PA arises and introduces additional states and steps in their description of PA. We here utilize mathematics to demonstrate that autoinhibitory conformational changes and the biasing of RAF to the nonautoinhibited state by RAF inhibitors is sufficient to drive PA. Additionally, we believe this is the critical missing step that can help explain PA and alleviate ongoing confusion in the field. Other processes, like negative allostery (or negative cooperativity) for inhibitor binding (18), 
preferential binding to the different RAF proteins and/or mutant RAF (2), altered dimerization affinity for drug-bound RAF proteins $(20,40,45)$, phosphorylation changes (28), scaffold proteins (29), allosteric trans-activation (24), and Ras nanoclusters (31) may all further tune the response to inhibitor, including in drug-specific manners.

With respect to 14-3-3 proteins, our mathematical analysis revealed that 14-3-3 protein overexpression could potentiate PA. This includes for agents that normally display minimal to no PA, like the third generation RAF inhibitors. Our experiments robustly detect PA to occur for a larger range of RAF inhibitor concentrations, consistent with our model. Our model also suggests that fold-change peak in PA signal can also increase, although we did not robustly detect such a change. There are several reasons that this may be. One is that our predictions focus on levels of active RAF kinase, but our experimental readout of RAF kinase activity is ERK phosphorylation. It is likely that the signal saturates, limiting the ability to measure fold changes. The ability to detect an increase in the range of drug concentrations that display PA would not be limited by saturation and would only require a monotonic relationship between RAF kinase activity and ERK phosphorylation, which seems reasonable to assume. 14-3-3proteins are promiscuous and pleiotropic, so it is possible that part of the effects observed with 14-3-3 transfection follow from other effects of 14-3-3 proteins; it is not possible to rule-out unknown alternative mechanisms involving 14-3-3 proteins. At minimum, our mathematical analysis yielded a novel hypothesis that led to the empirical observation that 14-3-3 protein amplification potentiates PA.

Additionally, our theoretical and experimental results highlight that previous thinking about PA was incomplete. The hypothesis that drugs that bind each protomer within a RAF dimer with similar affinity will be incapable of high-magnitude PA is here proven incorrect by our 14-3-3 overexpression experiments. Similarly, the pragmatic approach of finding inhibitors that do not display significant levels of PA in RAS mutant cells at the same concentrations that inhibit BRAF mutant signaling does not account for 14-3-3 abundance being a variable that can modulate the concentration at which PA occurs for a RAF mutant cell. Our work demonstrates how this happens. With the ability to drive increased levels of RAF signaling despite a RAF inhibitor being present, we hypothesize amplified expression of 14-3-3 proteins will emerge as a mechanism of resistance and toxicity should third generation RAF inhibitors make it to clinical use. 


\section{Materials and Methods}

\section{Mathematical Models and Analysis}

We focus on steady-state levels of the different states in which RAF can exist, as portrayed in the diagrams for each model. Between any two states an equilibrium relationship can be expressed as the ratio of abundances in the two states. Conservation of total protein quantities and zero value of total Gibbs free energy change at equilibrium both provide mechanisms to algebraically combine these expressions. We thereby derive algebraic expressions that relate the relative abundance of the RAF within its different monomeric and dimeric states. We perform algebraic manipulations and derive analytic solutions using Mathematica software (Wolfram Research). We perform numerical evaluations of these relationships, and generate plots of these equations using Python packages including numpy, scipy and matplotlib.

\section{Cell culture and transfection}

SK-MEL-2 cells were purchased from American Type Culture Collection (ATCC). SW48 cells with the G13D genoytype were obtained from Horizon Discovery. The DNA expression plasmid used for 14-3-3, 1481 pcDNA3 flag HA 14-3-3 zeta, was a gift from William Sellers (Addgene plasmid \# 9002). Cells were grown in EMEM (SK-MEL-2) or RPMI (SW48) containing $10 \%$ fetal bovine serum (FBS), penicillin $(100 \mathrm{U} / \mathrm{ml})$, streptomycin $(100 \mu \mathrm{g} / \mathrm{ml})$, and l-glutamine $(2 \mathrm{mM})$. Cells were cultured in $10 \mathrm{~cm}$ adherent culture dishes (VWR) and incubated at $37^{\circ} \mathrm{C}$ in $5 \% \mathrm{CO} 2$. At time point zero, cell media was changed to the cell line's respective media containing $10 \%$ fetal bovine serum (FBS) devoid of antibiotics. 24 hours later cells were transfected with empty packaged lipofectamine, or 5ug of 14-3-3-zeta expression plasmid DNA utilizing lipofectamine 2000 (Thermo Fisher Scientific) following manufacturers protocol. Cells were incubated for 24 hours and then were treated with RAF inhibitors at increasing doses for 2 hours. Cells were then prepared for Western blot analysis. All drugs were suspended and stored in DMSO, and all drug treatment groups carried the same amount of vehicle (DMSO). 


\section{Western blotting}

Cell lysates were generated using radioimmunoprecipitation assay buffer $[150 \mathrm{mM} \mathrm{NaCl}$, $1 \%$ NP-40, 0.5\% sodium deoxycholate, $0.1 \%$ SDS, $50 \mathrm{mM}$ tris ( $\mathrm{pH}$ 8.0)] containing protease and phosphatase inhibitor cocktail (Cell Signaling Technology) and incubated on ice for 1 hour, vortexing every five minutes. The total protein concentration was determined by Pierce Protein assay (Thermo Fisher Scientific). Protein samples $(5 \mu \mathrm{g})$ were resolved by electrophoresis on $12 \%$ SDS-polyacrylamide gels and electrophoretically transferred to polyvinylidene difluoride (PVDF) mem- branes (Millipore Corporation) for $20 \mathrm{~min}$ at $25 \mathrm{~V}$. The blots were probed with the mouse anti-phosphor-ERK antibody (675502, Biolegend) and rat anti-Total-ERK antibody (686902) overnight at 4 degrees Celsius. Blots were washed and probed with goat-anti-mouse Dylight 800 secondary antibody and goat-anti-rabbit AlexaFlour 680 antibody for 1 hour at room temperature. The protein bands were visualized using the Licor CLx Odyssey imaging station (Licor Biosystems). Comparative changes were measured with Licor Image Studio software.

\section{References}

1. F. Sanchez-Vega et al., Oncogenic Signaling Pathways in The Cancer Genome Atlas. Cell 173, 321-337 e310 (2018).

2. G. Bollag et al., Clinical efficacy of a RAF inhibitor needs broad target blockade in BRAF-mutant melanoma. Nature 467, 596-599 (2010).

3. C. Zhang et al., RAF inhibitors that evade paradoxical MAPK pathway activation. Nature 526, 583-586 (2015).

4. S. B. Peng et al., Inhibition of RAF Isoforms and Active Dimers by LY3009120 Leads to Anti-tumor Activities in RAS or BRAF Mutant Cancers. Cancer Cell 28, 384-398 (2015).

5. A. Nakamura et al., Antitumor activity of the selective pan-RAF inhibitor TAK-632 in BRAF inhibitor-resistant melanoma. Cancer Res 73, 7043-7055 (2013).

6. P. B. Chapman et al., Improved survival with vemurafenib in melanoma with BRAF V600E mutation. N Engl J Med 364, 2507-2516 (2011).

7. K. T. Flaherty et al., Combined BRAF and MEK inhibition in melanoma with BRAF V600 mutations. N Engl J Med 367, 1694-1703 (2012).

8. G. V. Long et al., Combined BRAF and MEK inhibition versus BRAF inhibition alone in melanoma. N Engl J Med 371, 1877-1888 (2014).

9. R. B. Corcoran et al., Combined BRAF and MEK Inhibition With Dabrafenib and Trametinib in BRAF V600-Mutant Colorectal Cancer. J Clin Oncol 33, 4023-4031 (2015).

10. R. B. Corcoran et al., Combined BRAF, EGFR, and MEK Inhibition in Patients with BRAF(V600E)-Mutant Colorectal Cancer. Cancer Discov 8, 428-443 (2018).

11. S. Kopetz et al., Encorafenib, Binimetinib, and Cetuximab in BRAF V600E-Mutated Colorectal Cancer. N Engl J Med 381, 1632-1643 (2019). 
12. V.Subbiah et al., Dabrafenib and Trametinib Treatment in Patients With Locally Advanced or Metastatic BRAF V600-Mutant Anaplastic Thyroid Cancer. J Clin Oncol 36, 7-13 (2018).

13. D. M. Hyman et al., Vemurafenib in Multiple Nonmelanoma Cancers with BRAF V600 Mutations. N Engl J Med 373, 726-736 (2015).

14. E. L. Diamond et al., Vemurafenib for BRAF V600-Mutant Erdheim-Chester Disease and Langerhans Cell Histiocytosis: Analysis of Data From the Histology-Independent, Phase 2, Open-label VE-BASKET Study. JAMA Oncol 4, 384-388 (2018).

15. S. J. Heidorn et al., Kinase-dead BRAF and oncogenic RAS cooperate to drive tumor progression through CRAF. Cell 140, 209-221 (2010).

16. P. I. Poulikakos, C. Zhang, G. Bollag, K. M. Shokat, N. Rosen, RAF inhibitors transactivate RAF dimers and ERK signalling in cells with wild-type BRAF. Nature 464, 427-430 (2010).

17. G. Hatzivassiliou et al., RAF inhibitors prime wild-type RAF to activate the MAPK pathway and enhance growth. Nature 464, 431-435 (2010).

18. Z. Karoulia et al., An Integrated Model of RAF Inhibitor Action Predicts Inhibitor Activity against Oncogenic BRAF Signaling. Cancer Cell 30, 485-498 (2016).

19. Z. Karoulia, E. Gavathiotis, P. I. Poulikakos, New perspectives for targeting RAF kinase in human cancer. Nat Rev Cancer 17, 676-691 (2017).

20. H. Lavoie et al., Inhibitors that stabilize a closed RAF kinase domain conformation induce dimerization. Nat Chem Biol 9, 428-436 (2013).

21. T. Jin et al., RAF inhibitors promote RAS-RAF interaction by allosterically disrupting RAF autoinhibition. Nat Commun 8, 1211 (2017).

22. C. K. Weber, J. R. Slupsky, H. A. Kalmes, U. R. Rapp, Active Ras induces heterodimerization of cRaf and BRaf. Cancer Res 61, 3595-3598 (2001).

23. Z. Yao et al., BRAF Mutants Evade ERK-Dependent Feedback by Different Mechanisms that Determine Their Sensitivity to Pharmacologic Inhibition. Cancer Cell 28, 370-383 (2015).

24. J. Hu et al., Allosteric activation of functionally asymmetric RAF kinase dimers. Cell 154, 1036-1046 (2013).

25. J. Yuan et al., The AMPK inhibitor overcomes the paradoxical effect of RAF inhibitors through blocking phospho-Ser-621 in the C terminus of CRAF. J Biol Chem 293, 1427614284 (2018).

26. H. Lavoie, M. Therrien, Regulation of RAF protein kinases in ERK signalling. Nat Rev Mol Cell Biol 16, 281-298 (2015).

27. C. Wellbrock, M. Karasarides, R. Marais, The RAF proteins take centre stage. Nat Rev Mol Cell Biol 5, 875-885 (2004).

28. M. Holderfield et al., RAF inhibitors activate the MAPK pathway by relieving inhibitory autophosphorylation. Cancer Cell 23, 594-602 (2013).

29. M. M. McKay, D. A. Ritt, D. K. Morrison, RAF inhibitor-induced KSR1/B-RAF binding and its effects on ERK cascade signaling. Curr Biol 21, 563-568 (2011).

30. Y. Kondo et al., Cryo-EM structure of a dimeric B-Raf:14-3-3 complex reveals asymmetry in the active sites of B-Raf kinases. Science 366, 109-115 (2019).

31. K. J. Cho et al., Raf inhibitors target ras spatiotemporal dynamics. Curr Biol 22, 945-955 (2012). 
32. R. E. Cutler, Jr., R. M. Stephens, M. R. Saracino, D. K. Morrison, Autoregulation of the Raf-1 serine/threonine kinase. Proc Natl Acad Sci U S A 95, 9214-9219 (1998).

33. M. Hekman et al., Dynamic changes in C-Raf phosphorylation and 14-3-3 protein binding in response to growth factor stimulation: differential roles of 14-3-3 protein binding sites. J Biol Chem 279, 14074-14086 (2004).

34. S. Rezaei Adariani et al., Structural snapshots of RAF kinase interactions. Biochem Soc Trans 46, 1393-1406 (2018).

35. E. Park et al., Architecture of autoinhibited and active BRAF-MEK1-14-3-3 complexes. Nature, (2019).

36. E. C. Stites, P. C. Trampont, Z. Ma, K. S. Ravichandran, Network analysis of oncogenic Ras activation in cancer. Science 318, 463-467 (2007).

37. E. C. Stites, P. C. Trampont, L. B. Haney, S. F. Walk, K. S. Ravichandran, Cooperation between Noncanonical Ras Network Mutations. Cell Rep 10, 307-316 (2015).

38. T. McFall et al., A systems mechanism for KRAS mutant allele-specific responses to targeted therapy. Sci Signal 12, (2019).

39. C. Wofsy, B. Goldstein, K. Lund, H. S. Wiley, Implications of epidermal growth factor (EGF) induced egf receptor aggregation. Biophys J 63, 98-110 (1992).

40. B. N. Kholodenko, Drug Resistance Resulting from Kinase Dimerization Is Rationalized by Thermodynamic Factors Describing Allosteric Inhibitor Effects. Cell Rep 12, 19391949 (2015).

41. E. Wilker, M. B. Yaffe, 14-3-3 Proteins--a focus on cancer and human disease. J Mol Cell Cardiol 37, 633-642 (2004).

42. A. J. Muslin, J. W. Tanner, P. M. Allen, A. S. Shaw, Interaction of 14-3-3 with signaling proteins is mediated by the recognition of phosphoserine. Cell 84, 889-897 (1996).

43. N. Dumaz, R. Marais, Protein kinase A blocks Raf-1 activity by stimulating 14-3-3 binding and blocking Raf-1 interaction with Ras. J Biol Chem 278, 29819-29823 (2003).

44. Z. Yao et al., RAF inhibitor PLX8394 selectively disrupts BRAF dimers and RASindependent BRAF-mutant-driven signaling. Nat Med 25, 284-291 (2019).

45. O. S. Rukhlenko et al., Dissecting RAF Inhibitor Resistance by Structure-based Modeling Reveals Ways to Overcome Oncogenic RAS Signaling. Cell Syst 7, 161-179 e114 (2018). 
A

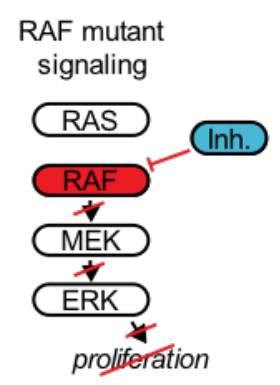

C

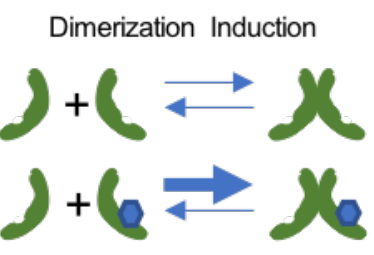

B
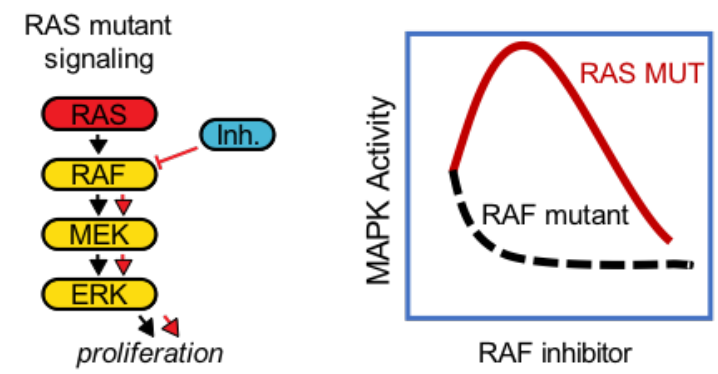

D Negative Cooperativity

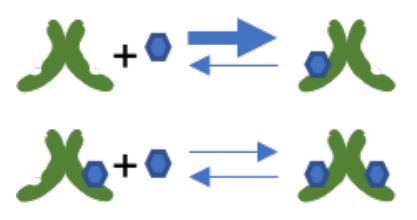

E

Conformational regulation and stabilization

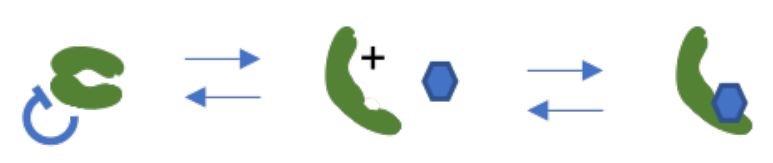

Figure 1. Paradoxical activation (PA) and possible mechanisms. (A) Schematic of the RAS/RAF/MEK/ERK signaling pathway. Intuition for this pathway originally posited that a RAF inhibitor would be able to inhibit signaling for both RAF and RAS mutant cancers. (B) Schematic of the PA concept. When a RAF inhibitor is given to a RAF mutant cell, there is a progressive decline in RAF signaling. However, when given to a RAS mutant cell, there is an increase in RAF signaling at lower doses of RAF inhibitor and suppression of RAF signaling at higher doses of inhibitor. (C, D) Proposed mechanisms of PA include drug-bound RAF having a higher dimerization affinity $(\mathrm{C})$ and negative cooperativity for drug binding in trans within a RAF dimer once one protomer has bound drug (D). (E) RAF autoinhibition and the stabilization of the non-autoinhibited, dimerization and signaling competent form of RAF by inhibitor is here considered as an alternative mechanism that may yield PA. 
A

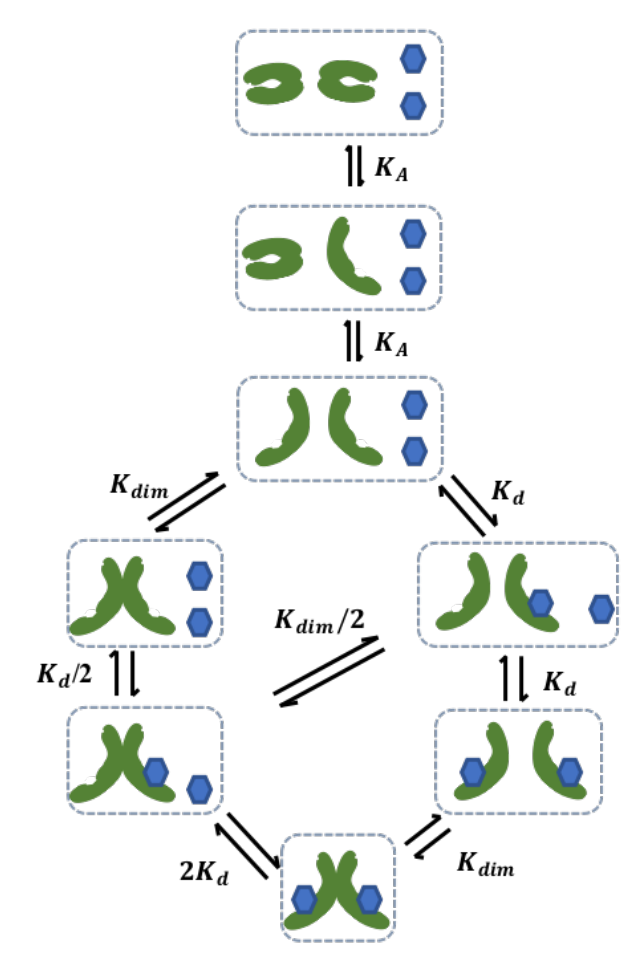

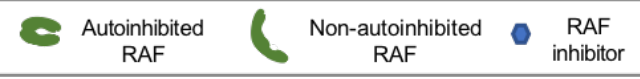

B

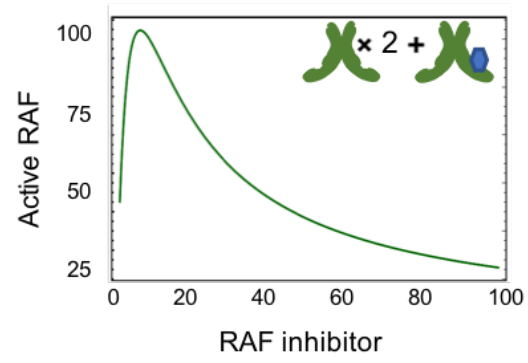

C
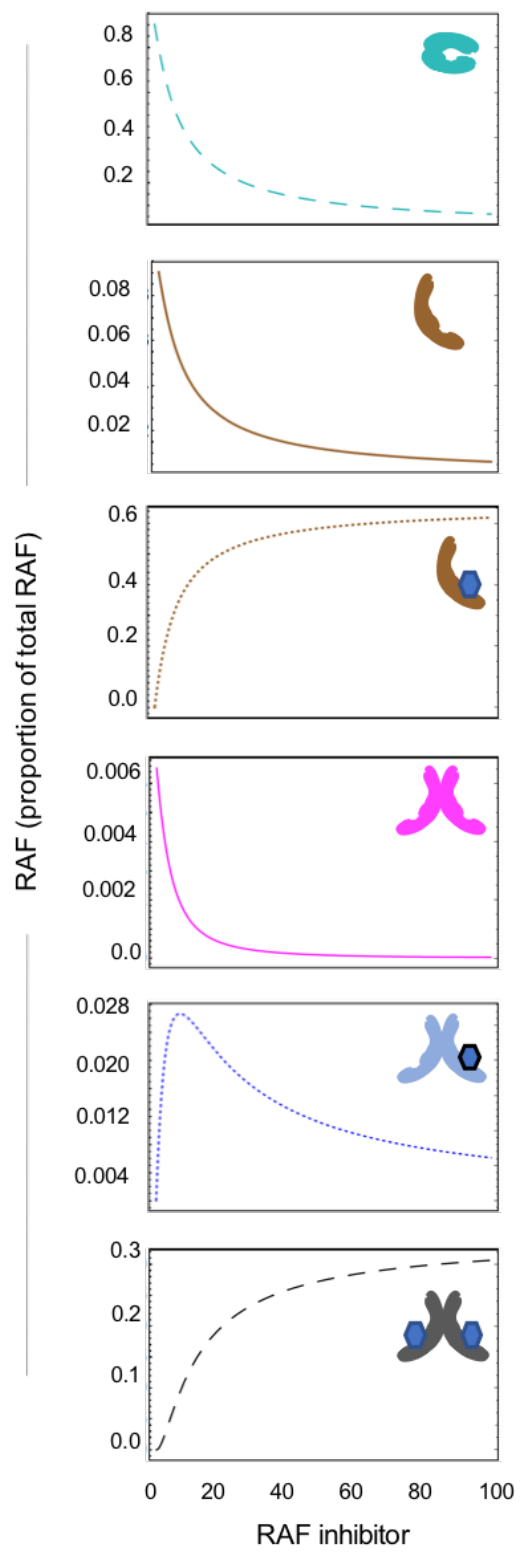

Figure 2. RAF autoinhibition is a mechanism that can produce paradoxical activation.

(A) Schematic of the RAF autoinhibition and dimerization model. (B) Representative plot demonstrating that this mechanism is sufficient to generate paradoxical activation. Plotted quantity is the number of active RAF protomers (within a dimer, not bound to drug) as a function of RAF inhibitor abundance, normalized to the maximum. (C) Representative plots portraying the proportion of total RAF in its different states to illuminate the signaling state changes underlying PA. In B and C, RAF inhibitor is the quantity of unbound drug relative to the affinity of the drug for a RAF monomer. 
A

Definitions of PA metrics

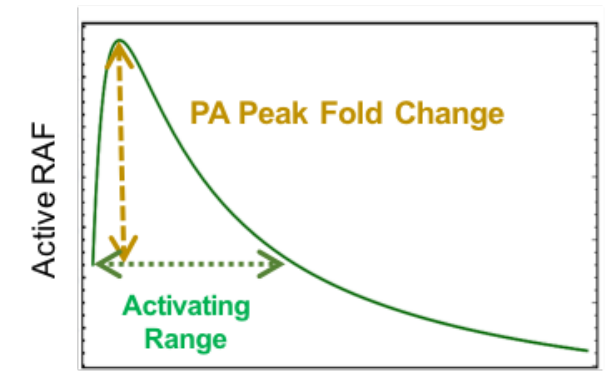

RAF inhibitor

C

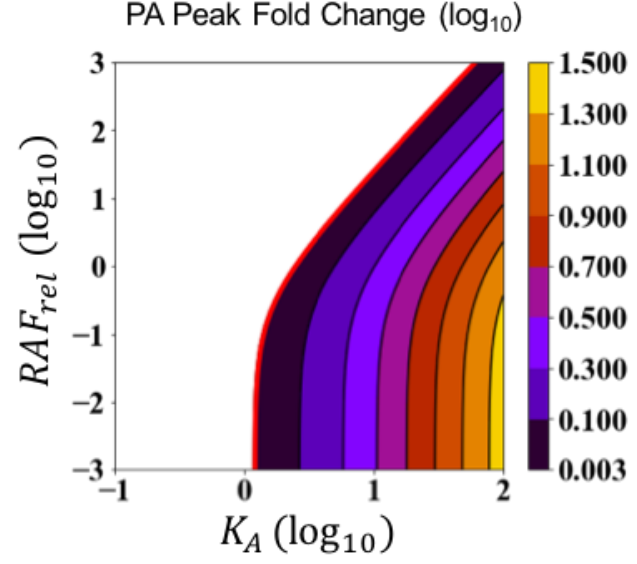

B

Definitions of PA parameters

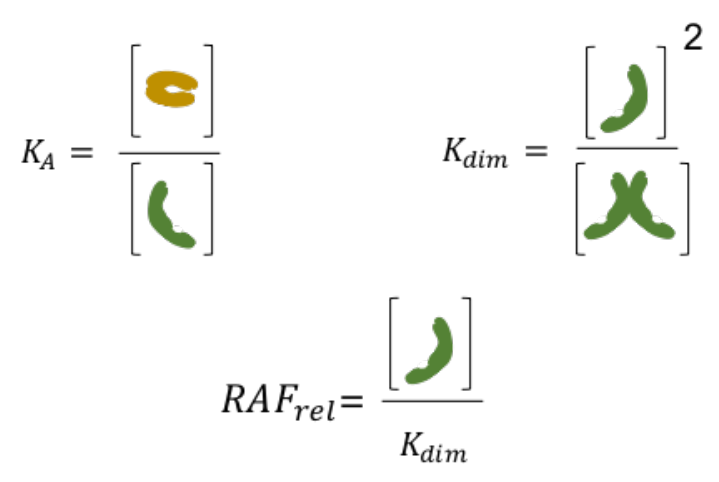

D

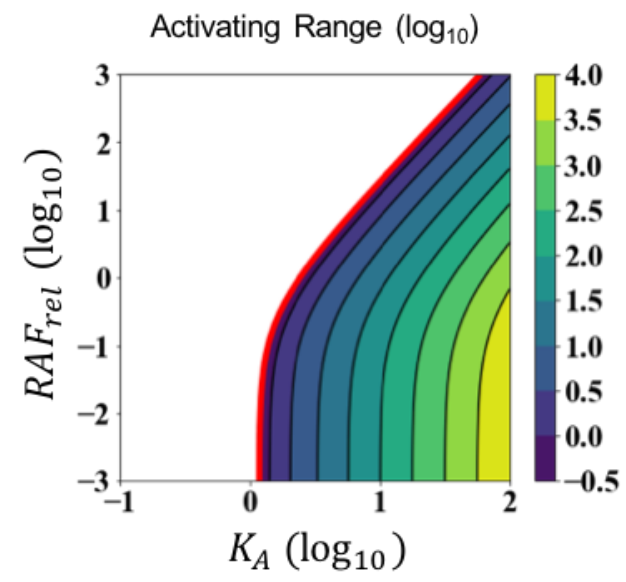

Figure 3. Magnitudes of paradoxical activation as a function of autoinhibition propensity

and RAF abundance. (A) Schematic to define two measures of PA: PA Peak Fold Change, which is the ratio of peak intensity to baseline intensity of active RAF protomers; and, PA Width, which is the drug concentration at which the inhibitory phase passes below the initial baseline relative to the affinity of the drug for a RAF monomer. (B) Schematic to define the key equilibrium constants considered here: $\mathrm{K}_{\mathrm{A}}$, or the autoinhibition equilibrium constant; $\mathrm{K}_{\mathrm{dim}}$, or the dimerization constant for $\mathrm{RAF}$, and $\mathrm{RAF}_{\text {rel }}$, or the quantity of RAF monomer relative to the dimerization constant. (C) Predicted PA Peak Fold Change for two key parameters of the autoinhibition model $\left(\mathrm{K}_{\mathrm{A}}\right.$ and $\left.\mathrm{RAF}_{\mathrm{rel}}\right)$, with magnitude of Fold Change presented as $\log _{10}$. Predicted PA Width for two key parameters of the model $\left(\mathrm{K}_{\mathrm{A}}\right.$ and $\left.\mathrm{RAF}_{\text {rel }}\right)$, with magnitude of PA Width as drug concentration relative to drug affinity presented in $\log _{10}$. 
A

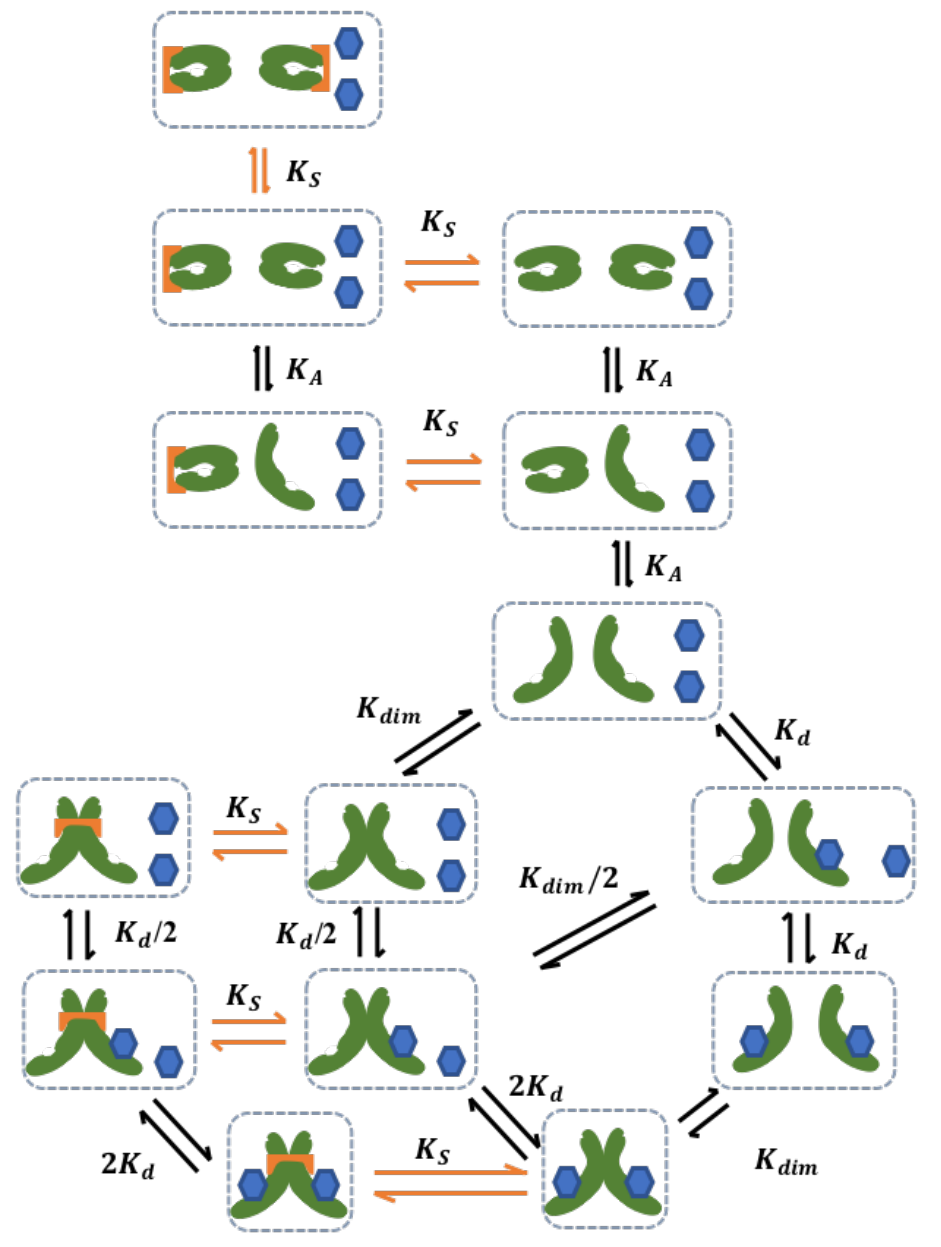

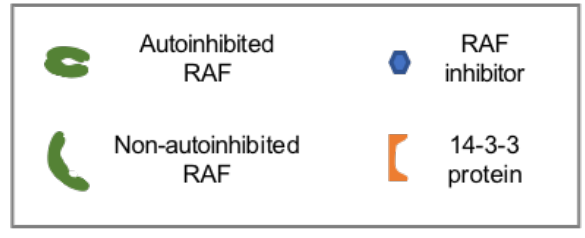

B
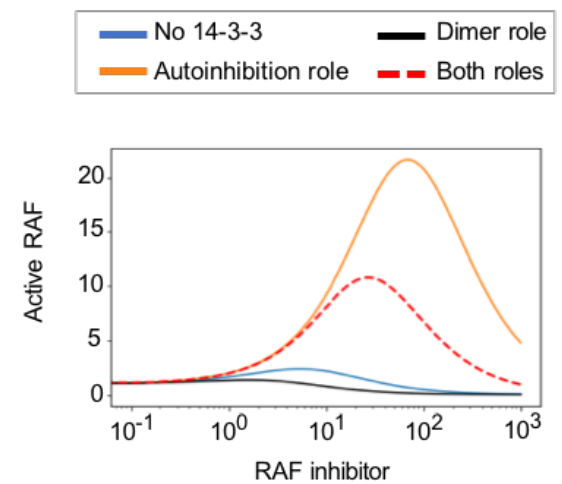

C
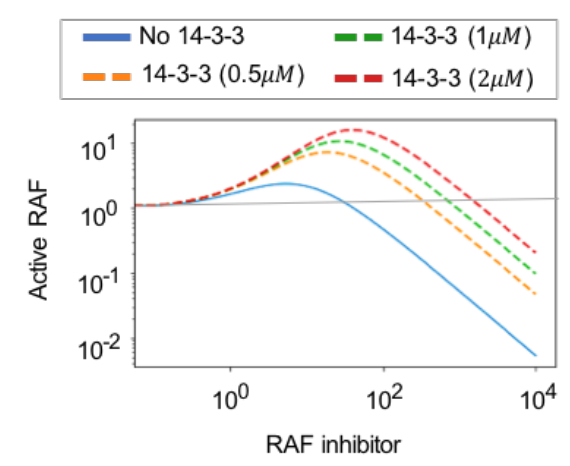

Figure 4. 14-3-3 proteins promote paradoxical activation by stabilizing the autoinhibited

form of RAF kinases. (A) Schematic of the modeled mechanism of 14-3-3 protein stabilization of the RAF autoinhibited form and the RAF dimer. (B) Predicted dose responses for the RAF autoinhibition model with 14-3-3 stabilizing (or not stabilizing) the autoinhibited form and/or the RAF dimer. (C) The model predicts that increasing levels of 14-3-3 protein will result in increased PA. In panels B and C, Active RAF is normalized to the level obtained with no RAF inhibitor, and RAF inhibitor is the quantity of unbound drug relative to the affinity of the drug for a RAF monomer. This is roughly proportional to total RAF inhibitor, and those interested should refer to the supplementary text for a more detailed description of nuances in the calculation. 
A

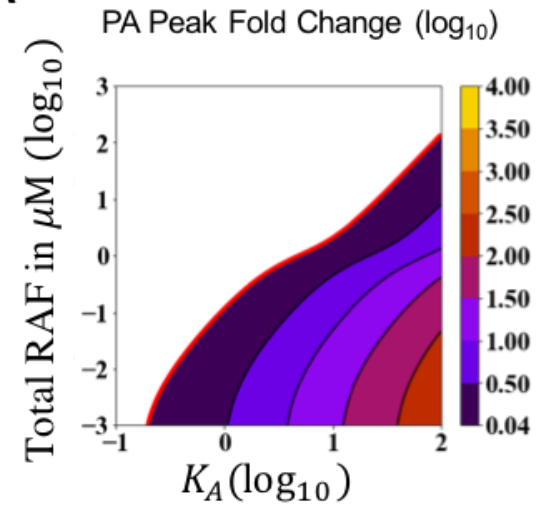

C

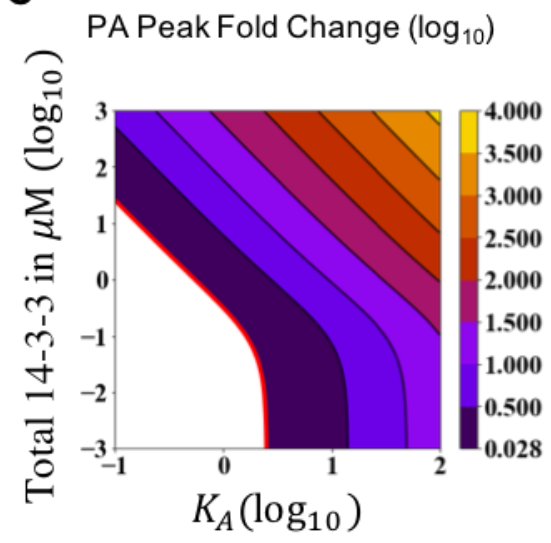

B

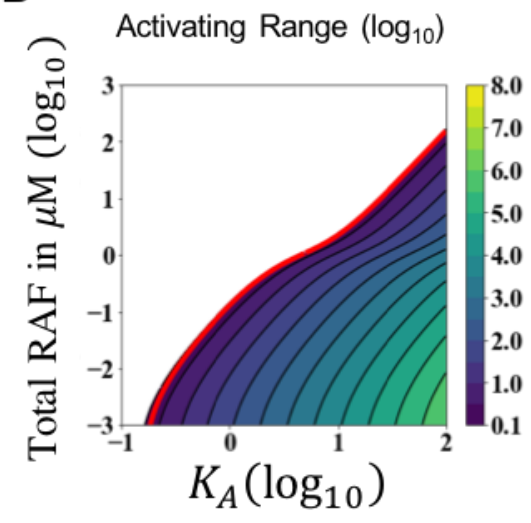

D

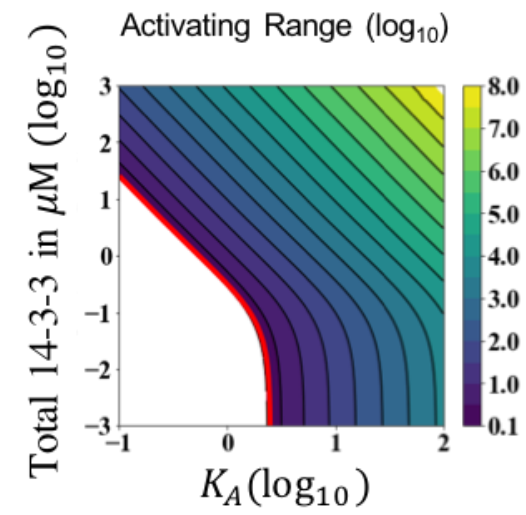

Figure 5. Magnitudes of paradoxical activation as a function of autoinhibition propensity, RAF abundance, and 14-3-3 abundance in a system with 14-3-3 proteins. (A) Numerical predictions for PA Peak Fold Change for two key parameters of the autoinhibition model $\left(\mathrm{K}_{\mathrm{A}}\right.$ and total RAF), with magnitude of Fold Change presented as $\log _{10}$. (B) Numerical predictions for PA Width for two key parameters of the model ( $\mathrm{K}_{\mathrm{A}}$ and total RAF), with magnitude of PA Width as drug concentration relative to drug affinity presented in $\log _{10}$. (C) Numerical predictions for PA Peak Fold Change for two key parameters of the autoinhibition model $\left(\mathrm{K}_{\mathrm{A}}\right.$ and total 14-3-3), with magnitude of Fold Change presented as $\log _{10}$. (B) Numerical predictions for PA Width for two key parameters of the model ( $\mathrm{K}_{\mathrm{A}}$ and total 14-3-3), with magnitude of PA Width as drug concentration relative to drug affinity presented in $\log _{10}$. 
A

\begin{tabular}{|c|c|c|}
\hline & \multicolumn{2}{|c|}{ Vemurafenib $(\mu \mathrm{M})$} \\
\hline & mock & $+14-3-3$ \\
\hline & 0.0. & $0 \quad 0^{\wedge} \times 0_{0}$ \\
\hline pERK & $-== \pm==$ & $====$ \\
\hline ERK & $=====$ & $==\equiv=$ \\
\hline
\end{tabular}

B

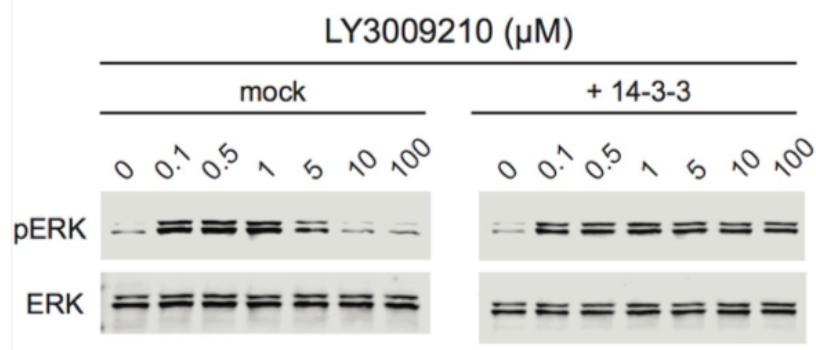

C

TAK-632 $(\mu \mathrm{M})$

\begin{tabular}{|c|c|c|}
\hline & mock & $+14-3-3$ \\
\hline & 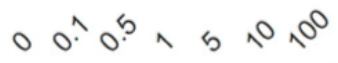 & $00^{\circ} 0^{5} \times 5 \times 0$ \\
\hline pERK & $===$ & $==\mathbf{=}==$ \\
\hline ERK & $======$ & $===\equiv==$ \\
\hline
\end{tabular}

D

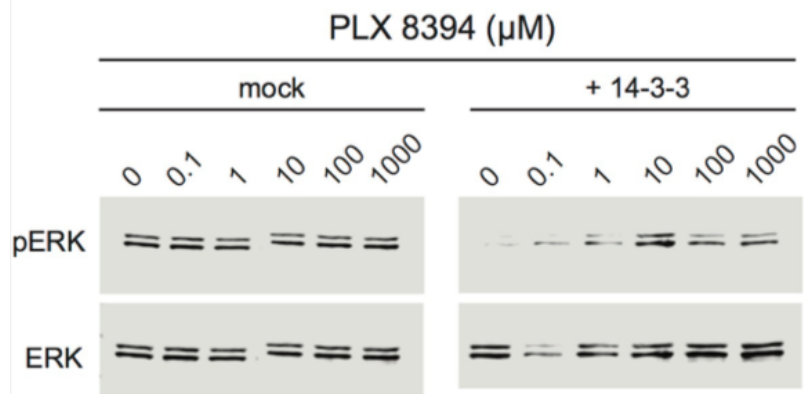

Figure 6. Increased 14-3-3 expression can potentiate paradoxical activation and lead to the breaking of "paradox breakers". Immunoblots of SK-MEL-2 cells that were transfected with 14-3-3 zeta or mock transfected. These transfected SK-MEL-2 cells were then treated with increasing doses of (A) vemurafenib, (B) LY3009210, (C) TAK-632, or (D) PLX3894. Results presented are representative of two independent experiments. 


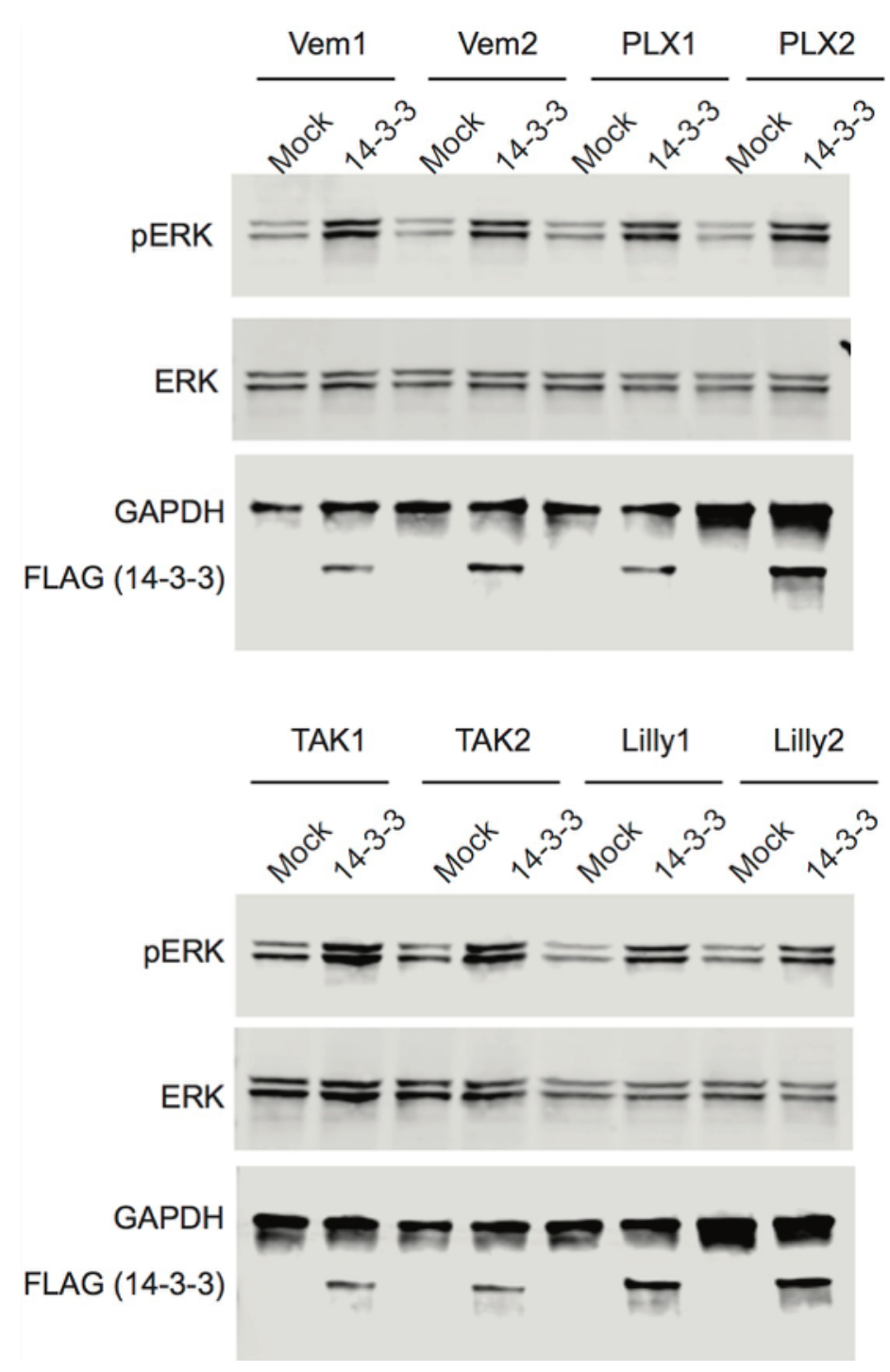

Figure S1. Immunoblots demonstrate 14-3-3 protein expression in 14-3-3 transfected cells. Immunoblots of SW48 cells that were mock transfected and transfected with14-3-3 for two replicates of the experiments with vemurafenib, PLX3894, LY3009210, and TAK-632. 


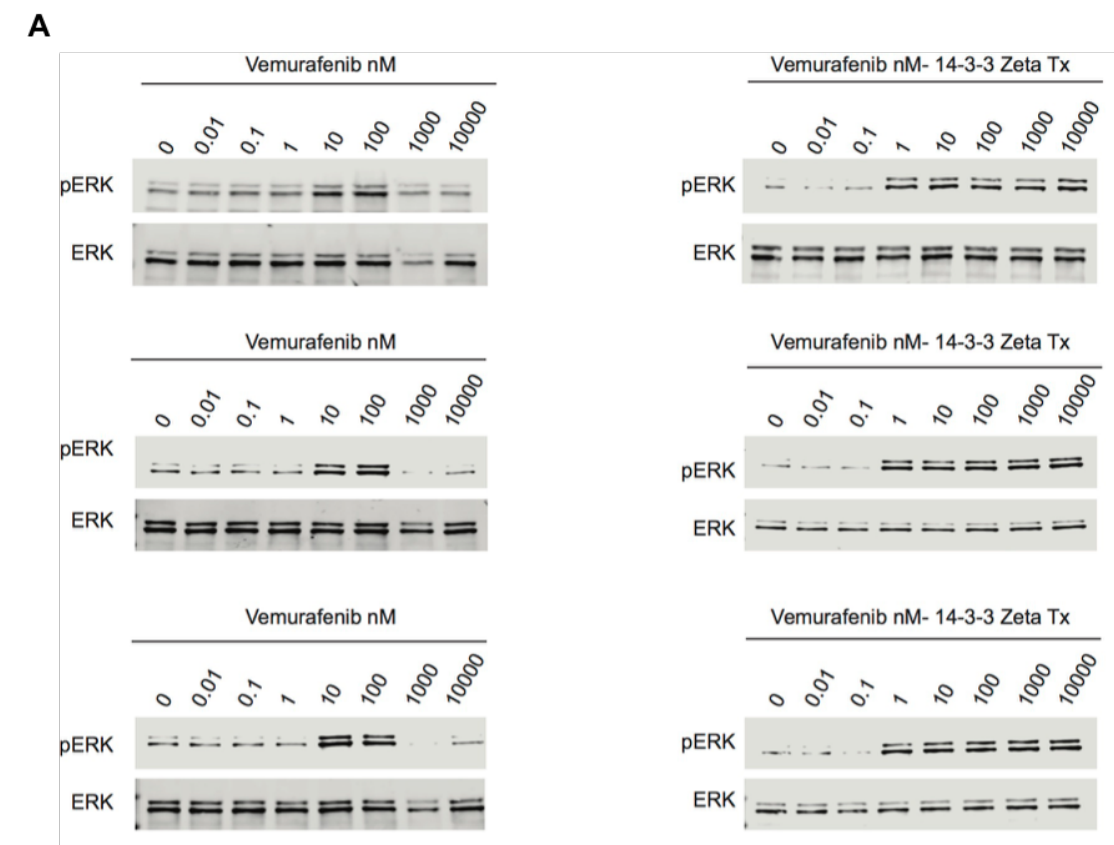

B

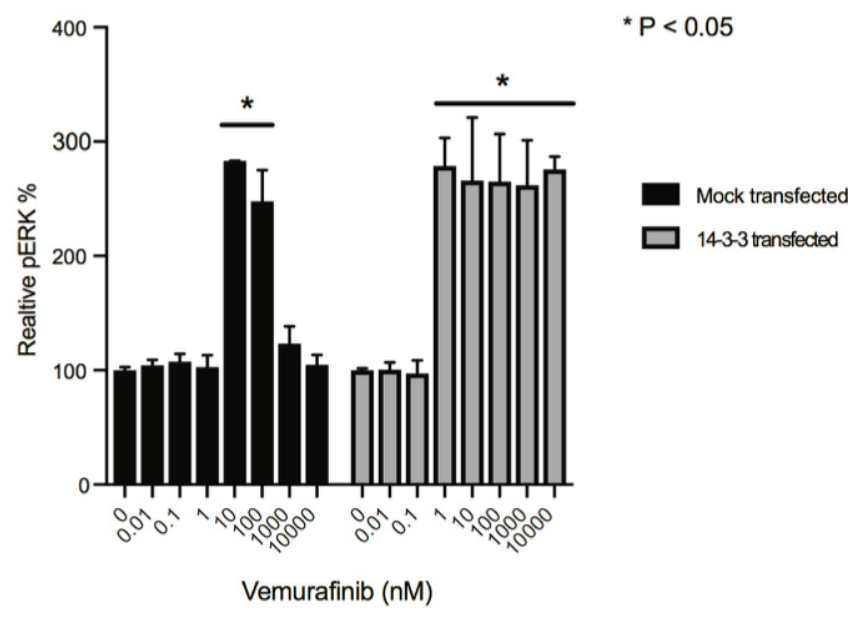

Figure S2. Increased 14-3-3 expression potentiates paradoxical activation in KRAS mutant colorectal cancer cells. (A) SW48 colorectal cancer isogenic derivatives with a KRAS G13D mutation were transfected with FLAG-tagged 14-3-3 zeta or mock transfected and then treated with increasing doses of vemurafenib. ERK phosphorylation and total ERK were then measured by immunoblotting. Results from three independent experiments are presented. (B) Average IR absorbance for pERK normalized to ERK from three independent experiments. Significant increases from the baseline value are indicated to demonstrate the range of concentrations where PA occurs. 